\title{
Cirugía de la región selar asistida por endoscopia
}

\author{
R. Prat; I. Galeano; J. Iñiesta*; R. Conde y J.A. Alvarez-Garijo
}

Servicios de Neurocirugía y de ORL*. Hospital Universitario La Fe. Valencia.

\section{Resumen}

Objetivos. El estudio pretende evaluar la utilidad del uso del endoscopio en la cirugía de la región selar a través del abordaje transesfenoidal transnasal en los adenomas hipofisarios y a través de abordajes mínimamente invasivos a la base de cráneo o el sistema ventricular en el caso de craneofaringiomas.

Material y métodos. Presentamos la experiencia preliminar en once casos intervenidos mediante cirugía asistida con endoscopia. Seis pacientes presentaban macroadenomas hipofisarios y fueron intervenidos por vía transesfenoidal transnasal. Cuatro pacientes presentaban craneofaringiomas, 2 de ellos recidivantes, que fueron abordados, 3 a través de un acceso supraciliar y uno mediante un abordaje transcortical transventricular, abordaje utilizado en un quiste supraselar intraventricular.

Resultados. Se consiguió la exéresis completa confirmada por RM de los adenomas hipofisarios en los que el uso del endoscopio con óptica de $30^{\circ}$ fue de utilidad en el control de la exéresis de los tumores con expansión supraselar. En el caso de los craneofaringiomas se alcanzó la exéresis completa en 3 de ellos uno de los cuales era recidivante, 2 por vía supraciliar y otro transcortical transventricular. En el caso restante, un craneofaringioma recidivante, la exéresis fue parcial por la íntima adherencia de la cápsula tumoral a las estructuras circundantes. En los 3 casos de acceso supraciliar, el endoscopio fue útil para el control de la exéresis del tumor localizado inferior al nervio óptico y la carótida interna ipsilaterales. En el acceso intraventricular el craneofaringioma que ocupaba el tercio anterior y medio del tercer ventrículo pudo resecarse a través del foramen de Monro, mediante una óptica de $30^{\circ}$ que permitió controlar y resecar el resto tumoral del tercio anterior. El quiste fue fenestrado.

Conclusiones. En cualquiera de las posibles vías de abordaje a la región selar, el uso de la cirugía asistida

Recibido: 1-12-07. Aceptado: 20-05-08 por endoscopia favorece una mayor radicalidad en la resección mediante el uso de abordajes mínimamente invasivos.

PALABRAS CLAVE: Endoscopia. Craneofaringioma. Adenoma hipofisario. Transesfenoidal. Ventricular.

Endoscopy-assisted surgery of the sellar region

Summary

Objective. To evaluate the usefulness of endoscopic assisted surgery of pituitary adenomas in transesphenoidal surgery, and in surgery of craneopharyngiomas using either minimally invasive approaches to the cranial base or transventricular approaches.

Material and methods. We present our preliminary experience in eleven patients operated of sellar region tumor by endoscopic assisted resection: 6 pituitary adenoma via transesphenoidal approach, 4 craneopharyngiomas 3 throung supraciliar approach and 1 by transcortical transventricular approach, and 1 suprasellar cyst.

Results. By using the 30 degrees optic the use of endoscope allowed complete resection, confirmed by postoperative MRI, of all six pituitary macroadenomas providing control of resection of supraselar remnants. Complete resection was achieved in three out of four craneopharyngiomas, 2 of them being recurrences. Three were operated by using a supraciliar approach to the cranial base and in one case transcortical transventricular resection of a recurrent intraventricular craneopharyngioma was performed. In the case with partial resection remnant were let in place due to the close adherence to peritumoral structures. In the three craneopharyngiomas operated via supraciliar approach endoscope allowed better control of inferior aspect of ipsilateral optic nerve and internal carotid artery. In the case of intraventricular 
craneopharyngioma, the use of $\mathbf{3 0}$ degrees endoscope provide control of resection of the anterior part of third ventricle through the foramen of Monro with no additional opening. The suprasellar cyst was fenestrated.

Conclusions. No matter which approach is going to be used in the resection of sellar tumors, endoscopy can play a crucial role in achieve complete resection with minimal morbidity by using minimally invasive procedures.

KEY WORDS: Endoscopy. Craneopharyngioma. Pituitary adenoma. Transesphenoidal. Ventricular

\section{Introducción}

El papel de la endoscopia en la biopsia y exéresis de lesiones intraventriculares ha quedado establecido en la literatura siendo las técnicas puramente endoscópicas y no la cirugía abierta de elección en numerosos procedimientos intraventriculares ${ }^{3,6,17}$.

Sin embargo el papel del endoscopio como instrumento óptico adicional a incluir en la batería de instrumentos utilizados en la cirugía abierta como el microscopio, el neuronavegador etc... queda por definir en las distintas regiones anatómicas y patologías principalmente cuando afectan a áreas de base de cráneo. Los tumores de la región selar son ese sentido paradigmáticos en cuanto a la complejidad anatómica de la región, la diversidad de su patología tumoral y la multiplicidad de opciones en su abordaje quirúrgico.

Nuestro estudio presenta la experiencia preliminar en el uso del endoscopio como asistente óptico en tumores de la región selar, que se intervinieron mediante procedimientos diversos pero que comparten la característica de las reducidas dimensiones del campo quirúrgico.

\section{Material y métodos}

Presentamos la experiencia preliminar en once casos intervenidos de forma consecutiva mediante cirugía asistida con endoscopia (Panoview Wolff de 2,7mm) de tumores de la región selar. Seis pacientes presentaban macroadenomas hipofisarios y fueron intervenidos por vía transesfenoidal transnasal. El tiempo transnasal fue llevado a cabo conjuntamente con el otorrinolaringólogo de referencia en patología nasal. En dos de los pacientes con adenomas hipofisarios se utilizó el neuronavegador (sistema BrainLab) como medio de posicionamiento, no utilizándose la radioscopia. La apertura del seno esfenoidal fue llevada a cabo con la ayuda del microscopio quirúrgico en todos los casos, mientras que la apertura del suelo de la silla turca y la apertura dural se realizó indistintamente bajo control microscópico o endoscópico. Cuando se con- sideró que se había alcanzado la máxima radicalidad con control de microscopio se introdujo en todos los casos el endoscopio para el control de restos no visualizables preferentemente supraselares. Cuatro pacientes presentaban craneofaringiomas, 2 de ellos recidivantes, que fueron abordados, 3 a través de un acceso subfrontal supraciliar y uno mediante un abordaje transcortical transventricular. El acceso subfrontal supraciliar condujo a la visualización y disección del plano aracnoideo con control de ambos nervios ópticos y carótidas internas, quiasma y complejo de la arteria comunicante anterior con lámina terminalis que se perforó en los 3 casos para completar la exéresis. En un caso la exéresis hubo de ser abandonada dada la íntima adherencia capsular al hipotálamo. Finalmente un paciente con un quiste supraselar con extensión intraventricular fue fenestrado endoscópicamente tras realizar una craneotomía frontal y posterior abordaje transcortical intraventricular.

Salvo en los pacientes intervenidos de adenomas hipofisarios y en el paciente con un quiste supraselar, que cuentan con RM a los 2 meses, en el resto de los pacientes se realizó RM de control antes de las 72 h que objetivó la extensión de la resección.

\section{Resultados}

La edad media de los pacientes fue de 52 años, 7 hombres y 4 mujeres. Se consiguió la exéresis completa confirmada por RM de los 6 adenomas hipofisarios en los que el uso del endoscopio con óptica de $30^{\circ}$ fue de utilidad en el control de la exéresis de los tumores con expansión supraselar. En el caso de los craneofaringiomas se alcanzó la exéresis completa en 3 de ellos uno de los cuales era recidivante, 2 por vía subfrontal supraciliar y otro también recidivante mediante un abordaje transcortical transventricular.

La exéresis fue parcial en el craneofaringioma recidivante intervenido mediante abordaje supraciliar subfrontal por la íntima adherencia de la cápsula tumoral a las estructuras circundantes. En los 3 casos de acceso supraciliar, el endoscopio fue útil para el control de la exéresis del tumor localizado inferior al nervio óptico y la carótida interna preferentemente ipsilaterales. La indemnidad del tallo hipofisario pudo ser confirmada durante la resección de forma nítida. En el acceso intraventricular el craneofaringioma que ocupaba el tercio anterior y medio del tercer ventriculo pudo resecarse a través del foramen de Monro en un primer tiempo de resección del tercio medio del tercer ventrículo mediante microscopio quirúrgico, y en un segundo tiempo mediante una óptica de $30^{\circ}$ que permitió controlar y resecar el resto tumoral del tercio anterior. El quiste fue fenestrado tras realización de un acceso transcortical transventricular y posteriormente utilizando el endoscopio de $0^{\circ}$. 


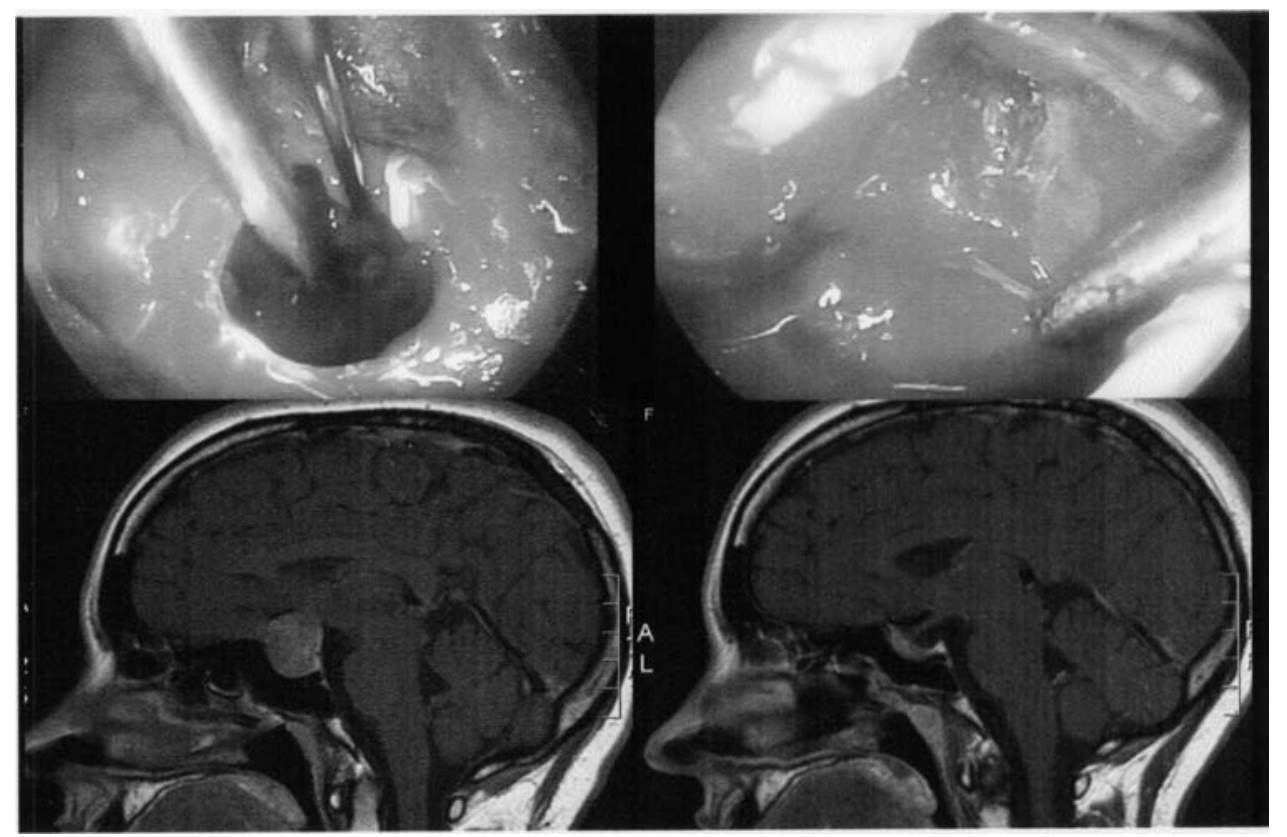

Figura 1. Imagen superior izquierda: imagen endoscópica durante la resección de macroadenoma hipofisario. Imagen superior derecha: imagen endoscópica intratumoral selar-supraselar. Imagen inferior izquierda: resonancia magnética preoperatoria mostrando macroadenoma hipofisario con extensión supraselar. Imagen inferior derecha: resonancia magnética postoperatoria

Presentamos a continuación de forma más detallada 3 casos representativos

\section{Caso 1 (figura 1)}

Paciente de 31 años de edad, con antecedente de fibroadenoma de mama intervenido y en tratamiento con acetato de ciproterona y etinilestradiol por alteraciones menstruales. Refiere trastorno subjetivo visual de 1 mes de evolución con cefalea rebelde a tratamiento médico. La exploración neurológica es normal presentando en el estudio campimétrico una hemianopsia bitemporal. La RM cerebral muestra una lesión tumoral selar con extensión supraselar que abomba a senos cavernosos. Rodea parcialmente carótidas intracavernosas sin estenosarlas y comprime quiasma óptico. El estudio hormonal muestra como datos más relevantes unos niveles de prolactina de $42.5 \mathrm{ng} / \mathrm{dl}$ y de factor de crecimiento insulínico tipo 1 de $777,7 \mathrm{U} / \mathrm{ml}$. Con el diagnóstico de macroadenoma hipofisario secretor de hormona de crecimiento es intervenida quirúrgicamente vía transnasal transesfenoidal, con uso combinado de microscopio quirúrgico y endoscopio de $2,7 \mathrm{~mm}$ con ópticas de $0^{\circ}$ y $30^{\circ}$. En este caso, se incluye la utilización de neuronavegador que sustituye a la radioscopia convencional. Durante la cirugía el control de posición

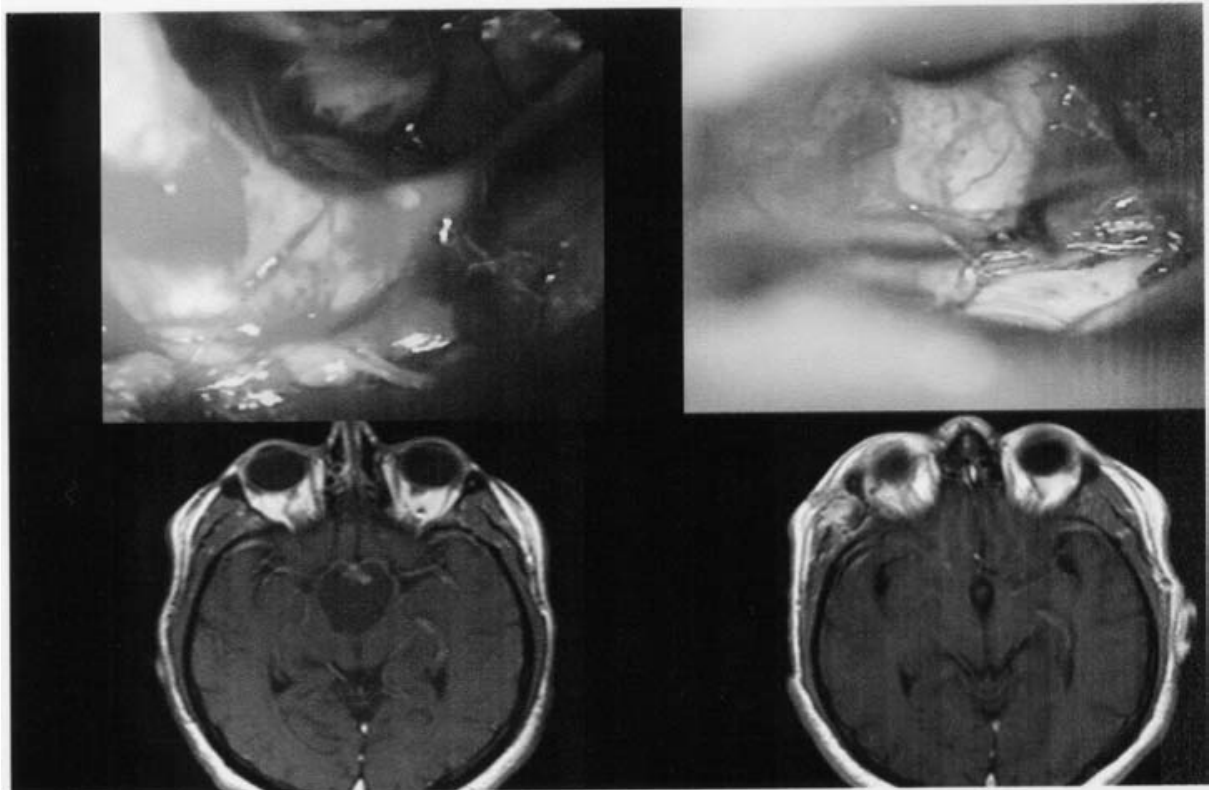

Figura 2: Imagen superior izquierda: imagen endoscópica a través de craneotomía supraorbitaria que muestra quiasma óptico y tallo hipofisario durante la resección tumoral. Imagen superior derecha: imagen con microscopio quirúrgico que muestra el quiasma óptico y el complejo de la arteria comunicante anterior en relación con la lámina terminalis abierta para completar la resección tumoral. Imagen inferior izquierda: resonancia magnética preoperatoria mostrando craneofaringioma quístico con nódulo intratumoral. Imagen inferior derecha: resonancia magnética postoperatoria. 


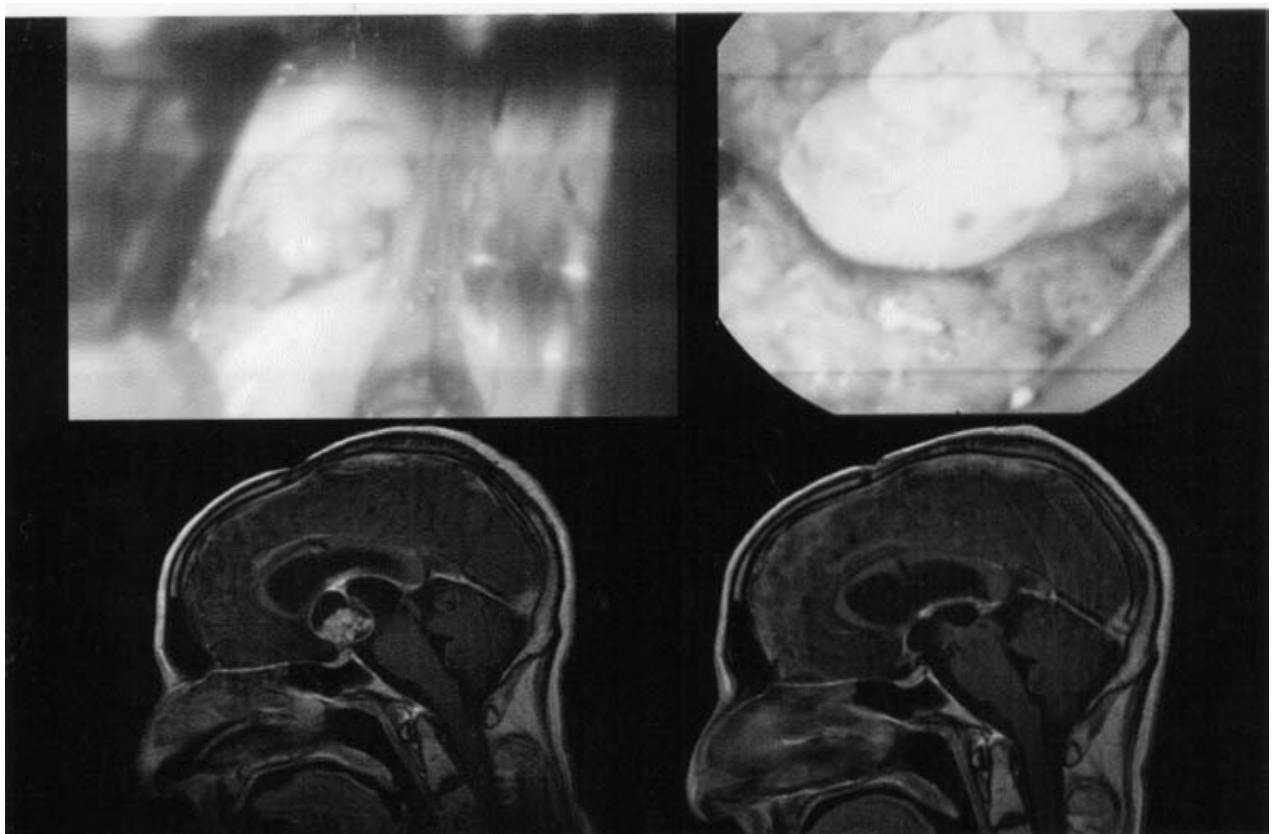

Figura 3: Imagen superior izquierda: imagen de microscopio quirúrgico en la que se aprecia craneofaringioma intraventricular que aflora a través de foramen de Monro. Imagen superior derecha: imagen endoscópica de parte de la lesión que se localiza en porción anterosuperior de tercer ventrículo. Imagen inferior izquierda: resonancia magnética preoperatoria mostrando craneofaringioma sólidoquístico intraventricular recidivante. Imagen inferior derecha: resonancia magnética postoperatoria.

por el neuronavegador en plano coronal permite un factor adicional de seguridad dada la relación del tumor con ambas carótidas. La paciente no presenta en el postoperatorio complicaciones locales y la función hormonal se normaliza en el control endocrinológico subsiguiente. Se le practica una exéresis tumoral completa confirmada en la RM a los 2 meses.

\section{Caso 2 (figura 2)}

Paciente de 38 años de edad que presenta cuadro de disminución de agudeza visual de 2 meses de evolución de forma progresiva que asocia cefalea y deterioro progresivo del nivel de consciencia. La RM cerebral muestra lesión selar supraselar expansiva solidoquística de 3,5 cm de diámetro máximo. Es intervenido mediante un abordaje subfrontal a través de una craneotomía supraciliar utilizando microscopio quirúrgico y endoscopio con ópticas de $0^{\circ}$ y $30^{\circ}$. Tras disección aracnoidea y visualización de ambos nervios ópticos, ambas carótidas y el complejo de la arteria comunicante anterior en relación con la lámina terminalis, se lleva a cabo exéresis completa de lesión compatible con craneofaringioma con apertura de lámina terminalis. El postoperatorio cursa sin complicaciones reseñables, confirmándose la exéresis completa en RM practicada a las $72 \mathrm{~h}$ de la cirugía.

\section{Caso 3 (figura 3)}

Paciente de 45 años de edad con antecedente de resección parcial extensa de craneofaringioma intraventricular un año antes. Con posterioridad presenta hidrocefalia que exigió de colocación de derivación ventrículo peritoneal. Experimenta cuadro de deterioro progresivo de funciones superiores con tetraespasticidad progresiva que mejora parcialmente con baclofeno. La RM cerebral de control muestra recidiva solidoquística con implantación en los tercios anterior y medio del tercer ventrículo. Se practica abordaje transcortical intraventricular aprovechando la misma craneotomía y abordaje que en la primera ocasión. El primer tiempo de la exéresis realizado con microscopio quirúrgico consigue la exéresis del tumor que aflora por el foramen de Monro y el localizado en el tercio medio del tercer ventrículo. La introducción del endoscopio de $30^{\circ}$ permite la visualización de resto de tumor en la porción más anterior del tercer ventrículo que se reseca bajo control endoscópico. Finalmente se lleva a cabo ventriculostomía del suelo del tercer ventrículo. La RM de control inmediata muestra exéresis completa de la tumoración. El paciente ha experimentado una evolución favorable con mejoría importante del cuadro de tetraespasticidad, que no obstante sigue precisando de tratamiento fisioterápico, y con normalización de sus funciones superiores.

\section{Discusión}

La cirugía de la región selar reúne por su complejidad anatómica y la potencial morbimortalidad asociada, las características propias de los procedimientos donde el control visual de todas las maniobras quirúrgicas es crítica. $\mathrm{Si}$ a este hecho añadimos la realización de procedimientos mínimamente invasivos, bien transesfenoidales, bien subfrontales, bien transventriculares en los que el campo quirúrgico es de mínimas dimensiones y se precisa de obte- 
ner de él el máximo rendimiento, se deduce que el papel de la endoscopia como asistente óptico puede ser de gran relevancia ${ }^{2,4,8,10,11,12,19}$.

En algunas series se refiere la utilidad relativa del uso del microscopio quirúrgico y del endoscopio en la cirugía hipofisaria vía transesfenoidal, confirmando una mayor tasa de exéresis con la introducción del endoscopio ${ }^{9,16}$, independientemente de su capacidad para reducir la morbilidad derivada de su menor invasividad quirúrgica ${ }^{2}$. En nuestra serie se aprecia un mayor rendimiento en la visualización de la porción supraselar de la tumoración mediante la óptica endoscópica de $30^{\circ}$. A pesar de que la apertura dural y la resección de la porción intraselar del tumor también se realizó con asistencia de endoscopia, no observamos que proporcione en estos pasos una ventaja adicional. Las mayores limitaciones del uso del endoscopio incluyen la disminución de la profundidad de campo, la necesidad de un reajuste manual prácticamente constante y la menor visibilidad en caso de sangrado ${ }^{7}$. Estas limitaciones son de menor entidad que en la cirugía endoscópica pura, puesto que el microscopio quirúrgico y la posibilidad de un control hemostático rápido y efectivo colaboran a facilitar la cirugía. En nuestra serie, y al contrario que en la cirugía endoscópica donde el sangrado es una complicación que obliga a prolongar el tiempo quirúrgico e incluso a abandonar la cirugía, ninguno de los procedimientos hubo de ser abandonado o se produjo un incremento del tiempo quirúrgico por la utilización del endoscopio. En dos casos apreciamos cómo la sustitución del control radioscópico por el uso del neuronavegador, permite, al presentar imágenes coronales que se corresponden con la anatomía quirúrgica, colaborar en la diferenciación del origen de un eventual sangrado y en el control de la exéresis reduciendo la exposición a radiaciones. En los abordajes endoscópicos transesfenoidales, el principal inconveniente en las diferentes series es el desarrollo de fístula de líquido cefalorraquídeo, dada la mínima invasividad en el abordaje pero la gran extensión de exéresis que puede alcanzarse con experiencias de tratamientos endoscópicos ampliados de lesiones supraselares que también incluyen lesiones prequiasmáticas como el tuberculum sellae o el planum esfenoidale $^{12}$. Sin embargo incluso en los procedimientos extendidos se han desarrollado técnicas para normalizar la incidencia de esta complicación ${ }^{12,20}$ que en nuestra serie no sufrió ningún paciente posiblemente en relación con la más limitada exéresis de estructuras óseas de la base de cráneo.

En la cirugía de craneofaringiomas el uso del endoscopio permite una mejor visualización de estructuras neurovasculares aumentado el porcentaje de resección aunque no minimiza las complicaciones endocrinológicas postquirúrgicas ${ }^{5,11,19}$. El uso del endoscopio permite abordajes mínimamente invasivos con máximas resecciones sin perder control de las estructuras paramediales de la base del cráneo y sin ser necesaria la realización de grandes e inestéticas incisiones o prolongadas retracciones cerebrales ${ }^{8}$. Su utilización no contraindica la realización de técnicas quirúrgicas o radioquirúrgicas adicionales mostrando su versatilidad en el manejo de la patología ${ }^{1}$. En nuestra serie la realización de un abordaje supraciliar subfrontal asistido con endoscopia permite un mayor control del campo quirúrgico, apreciándose la utilidad del uso del endoscopio de $30^{\circ}$ en la visualización del tallo hipofisario y de los restos tumorales en torno a los nervios ópticos y carótida interna principalmente en su aspecto inferior.

Los craneofaringiomas se localizan de forma infrecuente a nivel del tercer ventrículo y el tratamiento habitual es la realización de cirugía abierta con microcirugía, sin embargo, la utilización de técnicas endoscópicas se ofrece como más versátil y resolutiva ${ }^{14}$. El uso del endoscopio se concreta, bien colaborando en la exéresis completa de restos tumorales, (sin necesidad en muchos casos de ampliar el acceso a la porción anterior del tercer ventrículo con accesos que acarrean morbilidad), bien fenestrando, marsupializando o derivando el contenido de craneofaringiomas quísticos o sólido-quísticos ${ }^{4,15,18}$. En nuestro caso, la utilidad se aprecia en la posibilidad de realizar una técnica más segura incluso en el caso de tumores quísticos recidivantes, combinando la apertura de cavidades y su exploración, con la detección de restos tumorales en la porción anterior del tercer ventrículo deformado por la tumoración, y la posibilidad de realizar ventriculostomía asociada.

Las lesiones quísticas supraselares pueden ser abordadas mediante una variedad de técnicas quirúrgicas que sin embargo pueden ser dificultosas y estar asociadas a una alta morbilidad neurológica y endocrinológica. En nuestra serie, el quiste supraselar fue fenestrado tras realización de un acceso transcortical transventricular y posteriormente utilizando el endoscopio de $0^{\circ}$. La cirugía asistida en este caso no aporta ninguna ventaja adicional siendo de elección el acceso endoscópico puro que permite el mismo control visual con menor tiempo quirúrgico y morbilidad ${ }^{13}$.

En nuestra experiencia preliminar del uso de la cirugía endoscópica asistida en tumores de la región selar podemos concluir que en cualquiera de las posibles vías de abordaje a la región selar, la endoscopia favorece una mayor radicalidad en la resección mediante el uso de abordajes mínimamente invasivos. Este hecho no se asocia a una mayor morbimortalidad o incremento del tiempo quirúrgico de forma significativa.

\section{Bibliografía}

1. Barajas, M.A., Ramírez-Guzmán, G., RodríguezVázquez, C., et al.: Multimodal management of craniopharyngiomas: neuroendoscopy, microsurgery, and radiosurgery. J Neurosurg 2002; 97: 607-609. 
2. Cappabianca, P., Cavallo, L.M., Colao, A., et al.: Endoscopic endonasal transsphenoidal approach: outcome analysis of 100 consecutive procedures. Minim Invasive Neurosurg 2002; 45: 193-200.

3. Charalampaki, P., Filippi, R., Welschehold, S., Conrad, J., Perneckzy, A.: Tumors of the lateral and third ventricle: removal under endoscope-assisted keyhole conditions. Neurosurgery 2005; 57: 302-311.

4. Cinalli, J., Spennato, P., Cianciulli, E., Fiorillo, A., Di Maio, S., Maggi, G.: The role of transventricular neuroendoscopy in the management of craniopharyngiomas: three patient reports and review of the literature. J Pediatr Endocrinol Metabol 2006; 19: 341-354.

5. Frank, G., Pasquini, E., Doglietto, F., et al.: The endoscopic extended transsphenoidal approach for craniopharyngiomas. Neurosurgery 2006; 59: 75-83.

6. Gaab, M.R., Schroeder, H.W.S.: Neuroendoscopic approach to intraventricular lesions. J Neurosurg 1999; 88: 496-505.

7. Gondim, J., Schops, M., Tella, O.I. Jr.: Transnasal endoscopic surgery of the sellar region: study of the first 100 cases. Arq Neuropsiquiatr 2003; 61: 836-841

8. Jarrahy, R., Cha, S.T., Berci, G., Shahinian, H.K.: Endoscopic transglabellar approach to the anterior fossa and paranasal sinuses. J Craniofac Surg 2000; 11: 412-417.

9. Jho, H.D., Carrau, R.L.: Endoscopic endonasal transsphenoidal surgery: experience with 50 patients. J Neurosurg. 1997 ; 87: 44-51.

10. Kabil, M.S., Shahinian, H.K.: Application of the supraorbital endoscopic approach to tumors of the anterior cranial base. J Craniofac Surg 2005; 16: 1070-1074.

11. Kadri, H., Mawla, A.A.: Endoscopy-assisted microsurgical total resection of craniopharyngioma in childhood. Minim Invasive Neurosurg 2006; 49: 369-372.

12. Laufer, I., Anand, V.K., Schwartz, T.H.: Endoscopic, endonasal extended transsphenoidal, transplanum transtuberculum approach for resection of suprasellar lesions. J Neuro- surg 2007; 106: 400-406.

13. Nakahara, Y., Koga, H., Maeda, K., Takagi, M., Tabuchi, K.: Neuroendoscopic transventricular surgery for suprasellar cystic mass lesions such as cystic craniopharyngioma and Rathke cleft cyst. Neurol Med Chir 2004; 44: 408-413.

14. Nakamizo, A., Inamura, T., Nishio, S., Inoha, S., Ishibashi, H., Fukui, M.: Neuroendoscopic treatment of cystic craniopharyngioma in the third ventricle. Minim Invasive Neurosurg 2001; 44: 85-87.

15. Nicolato, A., Foroni, R., Rosta, L., Gerosa, M., Bricolo, A. : Multimodality stereotactic approach to the treatment of cystic craniopharyngiomas. Minim Invasive Neurosurg 2004; 47: 32-40.

16. Sethi, D.S., Pillay, P.K.: Endoscopic management of lesions of the sella turcica. J Laryngol Otol 1995; 109: 956962.

17. Soweidane, M., Luther, N.: Endoscopic resection of solid intraventricular tumors. J Neurosurg 2006; 105: 271278.

18. Tirakotai, W., Riegel, T., Schulte, D.M., Bertalanffy, H., Hellwig, D.: Neuroendoscopic stent procedure in obstructive hydrocephalus due to both foramina of monro occluding craniopharyngioma: technical note. Surg Neurol 2004; 61: 293-296.

19. Teo, C.: Application of endoscopy to the surgical management of craniopharyngiomas. Childs Nery Syst 2005; 21: 696-700.

20. Yano, S., Tsuiki, H., Kudo, M., et al.: Sellar repair with resorbable polyglactin acid sheet and fibrin glue in endoscopic endonasal transsphenoidal surgery. Surg Neurol 2007; 67: 59-64.

Prat, R.; Galeano, I.; Iñiesta, J.; Conde, R.; Alvarez-Garijo, J.A.: Cirugía de la región selar asistida por endoscopia. Neurocirugía 2008; 19: 501-506

Correspondencia postal: Dr. Ricardo Prat Acín. Paseo Alameda, 64. Esc B. $10^{\circ}$ - pta 47. 46023 Valencia. Spain. 